\title{
Knee Extensor and Flexor Strength are Related to One-Leg Hop Distances in Three Directions in Patients Six Months After Anterior Cruciate Ligament Reconstruction
}

Junya Aizawa ( $\boldsymbol{D}$ j.aizawa.ue@juntendo.ac.jp )

Juntendo University

Kenji Hirohata

Tokyo Medical and Dental University

Shunsuke Ohji

Tokyo Medical and Dental University

Takehiro Ohmi

Tokyo Medical and Dental University

Sho Mitomo

Tokyo Medical and Dental University

Hideyuki Koga

Tokyo Medical and Dental University

Kazuyoshi Yagishita

Tokyo Medical and Dental University

\section{Research Article}

Keywords: single-leg side jump, hamstring, quadriceps, ACL, reconstruction

Posted Date: November 25th, 2020

DOI: https://doi.org/10.21203/rs.3.rs-112315/v1

License: (c) (1) This work is licensed under a Creative Commons Attribution 4.0 International License. Read Full License

Version of Record: A version of this preprint was published at BMC Sports Science, Medicine and Rehabilitation on April 9th, 2021. See the published version at https://doi.org/10.1186/s13102-02100265-5. 


\section{Abstract}

Background: When planning rehabilitation and conditioning for performance enhancement and a return to sports after anterior cruciate ligament reconstruction, identifying the elements of physical function associated with one-leg hop is important. The purpose of this study was to clarify the relationship between one-leg hop distances in 3 directions and knee extensor and flexor strengths at 6 months after reconstruction.

Methods: Participants were 47 patients taking part in training sessions for sports involving cutting, pivoting, and jump-landing 6 months after reconstruction using a hamstring tendon. Demographics, activity scale score, days before and after reconstruction, and meniscus surgery procedure were analyzed. One-leg hop distances in 3 directions (anterior, lateral, and medial) and isokinetic strengths of knee extension and flexion were measured. Simple regression analyses using Spearman's rank correlation coefficient were performed to assess relationships between one-leg hop distances and knee strengths.

Results: In the involved limb, correlations between one-leg hop distance in 3 directions and knee strengths were significant and effect sizes ranged from medium to large. Correlation coefficients between all oneleg hop parameters and knee extension/flexion strengths at an angular velocity of $180^{\circ} / \mathrm{s}$ were greater than those of $60 \%$ s.

Conclusions: Knee strength showed moderate to high associations with lateral and medial one-leg hop distances in addition to anterior one-leg hop distance. The results of this study will be useful in planning and teaching conditioning to improve multi-directional one-legged hop skills and improve knee strength for a full-scale return to sports after reconstruction.

\section{Background}

Many athletes who damage the anterior cruciate ligament $(A C L)$ undergo reconstruction and long-term postoperative rehabilitation and conditioning to improve knee function and performance, often with a view to returning to their chosen sports ${ }^{1}$. However, after reconstruction, only $44-63 \%$ of athletes are able to return to the sports they were involved in before injury ${ }^{2}$. This represents a major issue for professionals involved in rehabilitation medicine and conditioning.

After reconstruction, decreases and asymmetry in one-leg hop (OLH) distance are likely to persist for a long time ${ }^{3-11}$. Decreased OLH distance is associated with lower improvements in subjective symptoms after reconstruction ${ }^{12}$, and hinders performance enhancement and a return to the sports the athlete was participating in prior to injury ${ }^{13-15}$. These issues should be considered, especially for athletes aiming to return to sports that require jump-landing and cutting. Identification of those elements of physical function associated with $\mathrm{OLH}$ is important when planning strength and conditioning training for performance enhancement and a return to sports after reconstruction. 
Previous studies have shown a moderate correlation between anterior OLH distance and knee extensor strength on the operated limb 6,16-18. Only the study by Sueyoshi et al. examined the relationship between anterior OLH distance and knee flexor strength in addition to knee extensor strength ${ }^{18}$. On the operated side in patients 6.6 months after reconstruction, they showed that the correlation coefficients between anterior OLH distance and peak torque at $180 \%$ s of knee flexion and extension were 0.58 and 0.42 , respectively ${ }^{18}$. In their study, 19 of 29 patients $(66 \%)$ underwent reconstruction with patellar tendon. Patterns of restoration of knee extensor and flexor strength differ between patients after reconstruction using patellar tendon and after reconstruction using hamstring graft ${ }^{19,20}$. Whether the results of the study by Sueyoshi et al., which suggest that knee flexor strength is more related to OLH distance than knee extensor strength, are directly applicable to patients after reconstruction using hamstring graft is thus unclear.

In sports that require frequent jump-landing and cutting, not only the anterior $\mathrm{OLH}$, but also lateral and medial OLH abilities are required. The relationship between anterior OLH distance and knee strength has been reported in several studies ${ }^{6,16-18}$. However, the relationship between lateral and medial OLH distances and knee strength has not been analyzed and is unknown.

The purpose of this study was to clarify the relationship between OLH distances for 3 directions and knee extensor/flexor strength for patients participating in a sport training session 6 months after reconstruction using hamstring graft. An additional aim was to provide useful information for conditioning plans regarding the return to sports 6 months after surgery. The 6-month time point was chosen because many orthopedic surgeons decide about clearance for returning to sports that involve jump-landing and cutting at this time ${ }^{21}$. We hypothesized that knee extensor and flexor strength would correlate with lateral and medial OLH distances in addition to anterior OLH.

\section{Methods}

\section{Participants}

From May 2015 to the end of July 2019, patients in this cross-sectional study were selected from among 276 patients who underwent $A C L$ reconstruction (Figure 1). Inclusion criteria were: primary/unilateral anatomical double-bundle reconstruction using either hamstring tendon autograft alone or gracilis tendon harvested in addition to hamstring tendon; age $\geq 16$ years and $\leq 40$ years at testing; postoperative rehabilitation with the same protocol used in the sports physical therapy department; participation in sports involving cutting, pivoting, and jump-landings (basketball, soccer, volleyball, badminton, tennis, or frisbee) before injury; and participating in the training sessions of the same sport played before surgery at approximately 6 months after reconstruction.

Patients were excluded if they had: past experience with ACL or meniscus injury or surgery on the other side; experienced injury that affected physical function in the lower back or lower limb; undergone reconstruction of other ligaments of the knee in addition to ACL; hoped to return to high-contact sports 
with tackling, such as football and rugby, martial arts sports that require pairing and throwing, such as judo and wrestling, striking sports such as boxing, and snow and ice sports such as skiing and ice hockey; did not go to the hospital or participate in sports for social reasons such as relocating or becoming pregnant after reconstruction; had comorbidities that hindered a return to sports; or had missing values. The institutional review board at our institution approved the study design (approval number: M2019-019). All participants provided written, informed consent.

Postoperative rehabilitation

The postoperative rehabilitation protocol was the same for all patients. However, patients who underwent repair of the middle posterior segment of the meniscus were prohibited from deep squatting until 3 months after surgery. Patients were permitted to begin isometric quadriceps exercises as tolerated from the day after ACL reconstruction. Using a knee brace (Straighten Position Knee-Joint Immobilizer; ALCARE, Tokyo, Japan) and crutches, partial weight-bearing (20 kg) was permitted on the first day after reconstruction, and gradually increased to the maximum body weight of each patient. Use of the knee brace and crutches was discontinued at 4 weeks after reconstruction. Range-of-motion exercises from full extension to $120^{\circ}$ of flexion were started on the second day after reconstruction. Closed kinetic chain exercises such as weight shifting and squatting were started 1-2 weeks after reconstruction. Open kinetic exercises such as seated knee extension using a machine load and a perturbation training program were started 3 months after reconstruction.

Running exercises were started in athletes who had cleared the criterion of limb symmetry index (LSI) of $65 \%$ of the knee extensor strength, as measured by the Biodex Multi-Joint Testing and Rehabilitation System (BDX-4; Biodex Medical Systems, New York, NY, USA) at 3 months after reconstruction. Speed and distance of running were gradually increased for joint effusion and symptoms of each patient. Once $80 \%$ of subjective full-speed running ability was achieved, athletic exercises related to the desired sporting activities were initiated with detailed instructions. All athletic exercises were specific to each patient, depending on the type of sport and position played. Participation in training sessions for the sports to which individual patients aimed to return was allowed from 6 months after reconstruction, as long as the patient showed no problematic symptoms in the joint and had sufficient knee isokinetic flexion/extension strength at $60 \%$ s ( $\mathrm{LSI}>80 \%)$ and had anterior OLH distance ( $\mathrm{LSI}>80 \%)$ after the specified athletic training had been completed 22,23 .

\section{Measurements}

Demographic characteristics, modified Tegner activity scale score before injury, days from injury to reconstruction, days after reconstruction, and meniscus surgery procedure were taken from medical records. OLH distances and knee strength in the operated (involved) limb and non-operated (uninvolved) limb were measured on the same day, which varied by patient. All physical function tests were conducted by 4 physiotherapists, each with more than 10 years of clinical experience in rehabilitation and conditioning of patients after ACL reconstruction. 
Sex was determined based on medical records. Height and weight were measured on the same testing day, and body mass index (BMI) was calculated. Oral questioning was used to confirm whether the knee that had undergone reconstruction was on the dominant side. The dominant limb was defined as the leg used to kick a ball to maximal distance ${ }^{24}$. The level of sports activity before injury was graded using the modified Tegner activity scale ${ }^{25}$.

The date of injury and date of reconstruction were confirmed by the patient and from medical records, and the number of days from date of injury to date of reconstruction was calculated. The number of days after reconstruction was the number of days from surgery to testing.

Meniscus injuries and treatments were confirmed from detailed records of arthroscopic findings during reconstruction. The injured segment (anterior, middle, or posterior), injury type (longitudinal, radial, or horizontal) and treatment method (suture, centralization, or partial meniscectomy) were confirmed. Participants were defined as being treated regardless of the method used.

OLH distances in the three directions (anterior, lateral, and medial) were measured in random order according to previous research 26,27 . Participants stood on one leg and were instructed to hop as far as possible and land on the same leg. The longest distance of 3 trials was recorded for each leg and each direction. The test was considered successful if the landing was stable. If the patient landed with early touchdown of the contralateral limb, which represented loss of balance, or took additional hops after landing, the hop was repeated. Patients were initially given a verbal description of the test, and were allowed to perform as many practice trials as desired, until they felt confident about the test. Three trials were performed for each leg, always starting with the non-surgical limb. For anterior OLH, the distance between the front end of the toe at starting position and the trailing edge of the heel at landing position was measured ${ }^{28}$. For lateral $\mathrm{OLH}$, the distance between the lateral side of the foot at starting position and the medial side of the foot at landing position was measured. For medial OLH, the distance between the medial side of the foot at starting position and the lateral side of the foot at landing position was measured. Results are represented as the distance-to-height ratio. Total OLH distance was calculated by standardizing the total of the three-direction OLH distances by height. Intraclass correlation coefficient (ICC) case 1 was calculated to examine the reproducibility of OLH distances in three directions in the involved limb and uninvolved limb of 10 athletes who met the same inclusion criteria as in this study. As a result, the ICCs of the single measurement values of the involved limb and uninvolved limb were in the range of $0.91-0.99$ and $0.91-0.96$, respectively, showing almost perfect reproducibility 29 .

The Biodex Multi-Joint Testing and Rehabilitation System was used to evaluate the isokinetic strength of the knee in extension/flexion. To minimize compensatory movements during testing, participants were seated and secured with padded straps around the thigh, pelvis, and torso. The femoral condyle of the tested limb was aligned with the rotation axis of the torque meter. Participants performed 3-5 repetitions of submaximal knee extension/flexion to familiarize themselves with the testing motion. To determine the strength of knee extension/flexion, participants performed 5 consecutive concentric contractions of extension/flexion at $60 \%$ s and $180 \%$ s. Peak torque within the 5 trials was extracted and normalized by 
body weight. The uninvolved limb was tested first. Results were represented by peak torque-to-weight ratio. The ICC of concentric peak torque of the knee using the Biodex system was close to 0.90 , indicating high to very high reproducibility ${ }^{30}$.

Statistical analysis

For sample size calculation, a priori power analysis and a previous study with similar outcome measurements revealed that at least 42 participants were required to achieve an alpha of 0.05 and a power of $0.90^{16,31}$. Due to potential attrition, a total of 47 subjects were recruited and tested in the current investigation. The normality of each variable was confirmed by the Shapiro-Wilk test. Simple regression analyses using Spearman's rank correlation coefficient were performed to clarify the relationship between OLH distance and knee strength. The magnitude of the effect size was determined to be strong for ${ }^{3} 0.50$ and medium for ${ }^{3} 0.30$ but $<0.50^{32}$. All data were analyzed with the Statistical Package for the Social Sciences for Windows (version 21.0; IBM Corp., New York, NY, USA). Values of P $<.05$ were used to indicate statistical significance.

\section{Results}

Table 1 shows the participant characteristics. Median age was 20 years and $68 \%$ were female. Median modified Tegner activity scale score before injury was 7.0. Mean time from reconstruction to testing was 185.0 days.

Table 2 shows values of OLH distance and knee strength. OLH distance-to-height ratio in the involved limb for the 3 directions ranged from $46.7 \%$ to $64.5 \%$. Total OLH distance of the involved limb and uninvolved limb were $165.6 \%$ and $188.1 \%$, respectively.

Table 3 shows bivariate correlation coefficients between variables in the involved limb and uninvolved limb. In the involved limb, correlations between OLH distance and knee strength were significant, and correlation coefficients were in the range of 0.48 to 0.65 . These effect sizes ranged from medium to large. In the uninvolved limb, correlations between OLH distance and knee strength were significant, and correlation coefficients were in the range of 0.51 to 0.62 ; these effect sizes were large. In the involved limb, the correlation coefficients between all OLH parameters and knee extension/flexion strength at an angular velocity of $180^{\circ} / \mathrm{s}$ were greater than those of $60^{\circ} \%$ s.

\section{Discussion}

This cross-sectional study analyzed associations between OLH distances for 3 directions and knee strength in patients participating in sports training sessions requiring jump-landing and cutting 6 months after reconstruction using hamstring graft. In this result, knee extension/flexion strength were moderately to strongly associated with lateral and medial OLH distances in addition to anterior OLH distance. 
In this study, in the involved limb, correlation coefficients between anterior OLH distance and knee extensor and flexor strength were in the range of 0.51 to 0.62 , and the relationships were large for both extension and flexion. Similar results were shown in the uninvolved limb. In the involved limb, knee strength at an angular velocity of $180^{\circ}$ /s was more closely related to anterior OLH distance than those of $60 \%$ s for both extension and flexion.

In a previous study of patients 6.6 months after reconstruction, the correlation coefficients of anterior OLH distance and knee extension/flexion strength at an angular velocity of $180 \%$ s were 0.42 and 0.58 , respectively, in the involved limb ${ }^{18}$. This result indicates that anterior OLH distance is more strongly associated with knee flexor strength than with extensor strength. In this study, correlation coefficients between anterior OLH distance and knee extensor and flexor strength at an angular velocity of $180^{\circ} / \mathrm{s}$ were 0.62 and 0.57 , respectively, and the relationships were similar. In the previous study, $66 \%$ of patients had undergone reconstruction using patellar tendon graft ${ }^{18}$. On the other hand, subjects in this study were only patients who underwent reconstruction with a hamstring graft. Postoperative symptoms and recovery patterns of knee extension/flexion strength differ depending on the type of graft used for reconstruction ${ }^{19,20}$. Therefore, the relationship between anterior OLH distance and knee extension/flexion strength may have differed between this and the previous study.

In a study of patients 8.3 months after reconstruction, the correlation coefficients of anterior OLH distance and knee extensor strength at angular velocities of $60 \%$ and $180 \%$ s were 0.36 and 0.59 , respectively, in the involved limbs ${ }^{16}$. Also in this study, in the involved limb, knee strength at an angular velocity of $180 \%$ s was more strongly related to the anterior OLH distance than that at $60 \% \mathrm{~s}$ for both extension and flexion. The results of this study supported the previous study.

In this study, the correlation coefficients between lateral OLH distance and knee extension/flexion strength at angular velocities of $60 \%$ s and $180 \%$ s were within the range of 0.52 to 0.65 in the involved limb. These effect sizes were large. The correlation coefficients between medial OLH distance and knee extension/flexion strength at angular velocities of $60 \%$ s and $180 \%$ s were within the range of $0.48-0.59$ in the involved limb, and these effect sizes were medium to large. Similar results were obtained with uninvolved limbs. In the involved limb, knee strength at an angular velocity of $180 \%$ s was more strongly related to lateral and medial OLH distances than those at $60 \% \mathrm{~s}$ for both extension and flexion. No previous reports have shown a relationship between lateral and medial OLH distance and knee strength in patients undergoing reconstruction.

In this study, the correlation coefficients between the total of anterior, lateral, and medial OLH distances and knee extension/flexion strength at angular velocities of $60 \%$ s and $180 \%$ s were within the range of 0.54-0.65 in the involved limb and these effect sizes were large. In the involved limb, knee strength at an angular velocity of $180 \%$ s correlated more strongly with total OLH distance than that at $60 \%$ sor both extension and flexion. The relationship between isokinetic knee extensor strength and vertical jump height of both legs at different angular velocities has been analyzed for healthy female basketball players ${ }^{33}$. In the previous study, isokinetic knee extensor strength at an angular velocity of $180^{\circ} / \mathrm{s}$ 
correlated more strongly with vertical jump height than that of $90^{\circ} / \mathrm{s}^{33}$. In this study, in the involved limb, the correlation coefficients between all OLH parameters and knee extension/flexion strength at an angular velocity of $180 \% \mathrm{~s}$ were greater than those of $60 \%$ s. One possible reason for this result is that the knee extension/flexion movements and muscle contraction speeds required by OLH are relatively close to those at an angular velocity of $180^{\circ} / \mathrm{s}$.

Previous studies have analyzed the relationship between anterior OLH distance and strength of both knee extension and flexion, selecting patients without limiting the type of graft used in reconstruction ${ }^{18}$. However, no previous studies have shown a relationship between three-way OLH distances, including lateral and medial, and knee extensor and flexor muscle strength, only for athletes who have undergone hamstring graft reconstruction. Rehabilitation and conditioning professions are required to plan instructional content after understanding the surgical information of the athlete and the characteristics of postoperative functional recovery. The results of this study provide evidence of conditioning guidance for athletes participating in training sessions 6 months after reconstruction with hamstring grafts, aiming to return to the sport in earnest. Specifically, to increase OLH distances in the three directions of anterior, lateral, and medial, measuring isokinetic knee extension/flexion strength at an angular velocity of $180 \% \mathrm{~s}$ and increasing these weight ratios is important.

Several reports have analyzed the relationship between LSI of anterior OLH distance and that of knee muscle strength in post-reconstruction patients $5,16,34,35$. Some of these studies concluded that the association between the two variables was either not significant or weak, and the results were inconsistent between studies. LSI is an index obtained by dividing the value of the involed limb by the value of the uninvoled limb. Since function of the non-operative side is reduced after reconstruction, if LSI is used as an index of functional recovery, knee strength and OLH ability of the reconstructive side may be overestimated ${ }^{36}$. The weight ratio, not the LSI of knee extension strength, is reportedly one of the factors that hinder the return to sports after reconstruction ${ }^{13}$. For these reasons, planning conditioning while checking the standardized value of knee muscle strength by body weight is important to improve $\mathrm{OLH}$ distance for returning to sports after reconstruction.

This study has some limitations. Since subjects in this study participated in sports that require frequent jump-landing and cutting, they were considered to have previously had relatively high one-legged hop skills in multiple directions. For this reason, there are limits to the applicability of the results to athletes who participate in sports that do not require much jump-landing and cutting. Values and relationships of OLH distance and knee strength may differ between dominant and non-dominant legs, but these were not analyzed separately ${ }^{37}$. Patterns of recovery for postoperative knee strength differ depending on whether the graft used in reconstruction is a hamstring or patellar tendon ${ }^{19,20}$. As this study targeted only patients after reconstruction with hamstring grafts, generalization of the findings to patients after reconstruction with patellar tendon graft remains limited.

\section{Conclusion}


In this cross-sectional study of patients who participated in sports training sessions that require jumplandings and cutting approximately 6 months after reconstruction using hamstring graft, both knee extensor and flexor strength were moderately to largely associated with lateral and medial OLH distances in addition to anterior OLH distance. The results of this study will be useful in planning and teaching conditioning to improve multi-directional OLH skill and strengthen knee strength for a full-scale return to the sport 6 months after reconstruction.

\section{Abbreviations}

Anterior cruciate ligament (ACL)

One-leg hop (OLH)

Limb symmetry index (LSI)

Intraclass correlation coefficient (ICC)

\section{Declarations}

Ethics approval and consent to participate: The Institutional Review Board at Tokyo Medical and Dental University approved the study design (approval number: M2019-019). The study was conducted in accordance with the Declaration of Helsinki. All participants provided written, informed consent.

Consent for publication: Not applicable

Availability of data and materials: The datasets used and/or analysed during the current study are available from the corresponding author on reasonable request.

Competing interests: The authors certify that there are no conflicts of interest with any financial organization regarding the material discussed in the manuscript.

Funding: The authors report no involvement in the research by the sponsor that could have influenced the outcome of this work.

Authors' contributions: Junya Aizawa and Kenji Hirohata contributed substantially to the conception or design of the manuscript; Junya Aizawa, Shunsuke Ohji, Takehiro Ohmi, and Sho Mitomo contributed substantially to the acquisition, analysis and interpretation of the data. All authors participated in drafting the manuscript. Hideyuki Koga and Kazuyoshi Yagishita revised the manuscript critically. All authors contributed equally to the manuscript and read and approved the final version of the manuscript.

Acknowledgements: Not applicable

\section{References}


1. Lai CCH, CL Ardern, JA Feller, KE Webster. Eighty-three per cent of elite athletes return to preinjury sport after anterior cruciate ligament reconstruction: a systematic review with meta-analysis of return to sport rates, graft rupture rates and performance outcomes. Br J Sports Med 2018;52(2):128-38.

2. Ardern CL, KE Webster, NF Taylor, JA Feller. Return to sport following anterior cruciate ligament reconstruction surgery: a systematic review and meta-analysis of the state of play. Br J Sports Med 2011;45(7):596-606.

3. Ageberg E, R Thomeé, C Neeter, KG Silbernagel, EM Roos. Muscle strength and functional performance in patients with anterior cruciate ligament injury treated with training and surgical reconstruction or training only: a two to five-year followup. Arthritis Rheum 2008;59(12):1773-9.

4. Beischer S, E Hamrin Senorski, C Thomeé, K Samuelsson, R Thomeé. Correction to: Young athletes return too early to knee-strenuous sport, without acceptable knee function after anterior cruciate ligament reconstruction. Knee Surg Sports Traumatol Arthrosc 2018;26(5):1586.

5. Keays SL, JE Bullock-Saxton, P Newcombe, AC Keays. The relationship between knee strength and functional stability before and after anterior cruciate ligament reconstruction. J Orthop Res 2003;21(2):231-7.

6. Petschnig R, R Baron, M Albrecht. The relationship between isokinetic quadriceps strength test and hop tests for distance and one-legged vertical jump test following anterior cruciate ligament reconstruction. J Orthop Sports Phys Ther 1998;28(1):23-31.

7. Schmitt LC, MV Paterno, KR Ford, GD Myer, TE Hewett. Strength Asymmetry and Landing Mechanics at Return to Sport after Anterior Cruciate Ligament Reconstruction. Med Sci Sports Exerc 2015;47(7):1426-34.

8. Schmitt LC, MV Paterno, TE Hewett. The impact of quadriceps femoris strength asymmetry on functional performance at return to sport following anterior cruciate ligament reconstruction. $J$ Orthop Sports Phys Ther 2012;42(9):750-9.

9. Thomeé R, C Neeter, A Gustavsson, P Thomeé, J Augustsson, B Eriksson, et al. Variability in leg muscle power and hop performance after anterior cruciate ligament reconstruction. Knee Surg Sports Traumatol Arthrosc 2012;20(6):1143-51.

10. Toole AR, MP Ithurburn, MJ Rauh, TE Hewett, MV Paterno, LC Schmitt. Young Athletes Cleared for Sports Participation After Anterior Cruciate Ligament Reconstruction: How Many Actually Meet Recommended Return-to-Sport Criterion Cutoffs? J Orthop Sports Phys Ther 2017;47(11):825-33.

11. Raoul T, S Klouche, B Guerrier, B El-Hariri, S Herman, A Gerometta, et al. Are athletes able to resume sport at six-month mean follow-up after anterior cruciate ligament reconstruction? Prospective functional and psychological assessment from the French Anterior Cruciate Ligament Study (FAST) cohort. Knee 2019;26(1):155-64.

12. Cristiani R, C Mikkelsen, G Edman, M Forssblad, B Engström, A Stålman. Age, gender, quadriceps strength and hop test performance are the most important factors affecting the achievement of a patient-acceptable symptom state after ACL reconstruction. Knee Surg Sports Traumatol Arthrosc 2020;28(2):369-80. 
13. Lentz TA, G Zeppieri, SM Tillman, PA Indelicato, MW Moser, SZ George, et al. Return to preinjury sports participation following anterior cruciate ligament reconstruction: contributions of demographic, knee impairment, and self-report measures. J Orthop Sports Phys Ther 2012;42(11):893-901.

14. Lentz TA, G Zeppieri, SZ George, SM Tillman, MW Moser, KW Farmer, et al. Comparison of physical impairment, functional, and psychosocial measures based on fear of reinjury/lack of confidence and return-to-sport status after ACL reconstruction. Am J Sports Med 2015;43(2):345-53.

15. Grindem H, L Snyder-Mackler, H Moksnes, L Engebretsen, MA Risberg. Simple decision rules can reduce reinjury risk by $84 \%$ after $A C L$ reconstruction: the Delaware-Oslo $A C L$ cohort study. $\mathrm{Br} J$ Sports Med 2016;50(13):804-8.

16. Nagai T, ND Schilaty, ER Laskowski, TE Hewett. Hop tests can result in higher limb symmetry index values than isokinetic strength and leg press tests in patients following ACL reconstruction. Knee Surg Sports Traumatol Arthrosc 2020;28(3):816-22.

17. Wilk KE, WT Romaniello, SM Soscia, CA Arrigo, JR Andrews. The relationship between subjective knee scores, isokinetic testing, and functional testing in the ACL-reconstructed knee. J Orthop Sports Phys Ther 1994;20(2):60-73.

18. Sueyoshi T, A Nakahata, G Emoto, T Yuasa. Single-leg hop test performance and isokinetic knee strength after anterior cruciate ligament reconstruction in athletes. Orthop J Sports Med 2017;5(11):2325967117739811.

19. Tan SHS, BPH Lau, L Krishna. Outcomes of anterior cruciate ligament reconstruction in females using patellar-tendon-bone versus hamstring autografts: a systematic review and meta-analysis. $J$ Knee Surg 2019;32(8):770-87.

20. Machado F, P Debieux, CC Kaleka, D Astur, MS Peccin, M Cohen. Knee isokinetic performance following anterior cruciate ligament reconstruction: patellar tendon versus hamstrings graft. Phys Sportsmed 2018;46(1):30-5.

21. Müller U, M Krüger-Franke, M Schmidt, B Rosemeyer. Predictive parameters for return to pre-injury level of sport 6 months following anterior cruciate ligament reconstruction surgery. Knee Surg Sports Traumatol Arthrosc 2015;23(12):3623-31.

22. Koga $H, T$ Muneta, $K$ Yagishita, $T$ Watanabe, $T$ Mochizuki, $M$ Horie, et al. Mid- to long-term results of single-bundle versus double-bundle anterior cruciate ligament reconstruction: randomized controlled trial. Arthroscopy 2015;31(1):69-76.

23. Muneta T, H Koga, T Mochizuki, YJ Ju, K Hara, A Nimura, et al. A prospective randomized study of 4strand semitendinosus tendon anterior cruciate ligament reconstruction comparing single-bundle and double-bundle techniques. Arthroscopy 2007;23(6):618-28.

24. Aizawa J, K Hirohata, $\mathrm{S}$ Ohji, $\mathrm{T}$ Ohmi, $\mathrm{K}$ Yagishita. Limb-dominance and gender differences in the ground reaction force during single-leg lateral jump-landings. J Phys Ther Sci 2018;30(3):387-92.

25. Fältström A, M Hägglund, J Kvist. Patient-reported knee function, quality of life, and activity level after bilateral anterior cruciate ligament injuries. Am J Sports Med 2013;41(12):2805-13. 
26. Gustavsson A, C Neeter, P Thomeé, KG Silbernagel, J Augustsson, R Thomeé, et al. A test battery for evaluating hop performance in patients with an $A C L$ injury and patients who have undergone $A C L$ reconstruction. Knee Surg Sports Traumatol Arthrosc 2006;14(8):778-88.

27. Aizawa J, K Hirohata, S Ohji, T Ohmi, H Koga, K Yagishita. Factors associated with psychological readiness to return to sports with cutting, pivoting, and jump-landings after primary anterior cruciate ligament reconstruction. Orthop J Sports Med 2020; doi: https://doi.org/10.1177/2325967120964484.

28. Munro AG, LC Herrington. Between-session reliability of four hop tests and the agility T-test. J Strength Cond Res 2011;25(5):1470-7.

29. Landis JR, GG Koch. The measurement of observer agreement for categorical data. Biometrics 1977;33(1):159-74.

30. de Araujo Ribeiro Alvares JB, R Rodrigues, R de Azevedo Franke, BG da Silva, RS Pinto, MA Vaz, et al. Inter-machine reliability of the Biodex and Cybex isokinetic dynamometers for knee flexor/extensor isometric, concentric and eccentric tests. Phys Ther Sport 2015;16(1):59-65.

31. Kanda Y. Investigation of the freely available easy-to-use software 'EZR' for medical statistics. Bone Marrow Transplant 2013;48(3):452-8.

32. Cohen J. Statistical power analysis for the behavioral sciences. 2nd ed. Hillsdale, NJ: Lawrence Erlbaum; 1988.

33. Rouis M, L Coudrat, H Jaafar, JR Filliard, H Vandewalle, Y Barthelemy, et al. Assessment of isokinetic knee strength in elite young female basketball players: correlation with vertical jump. J Sports Med Phys Fitness 2015;55(12):1502-8.

34. Barfod KW, JA Feller, T Hartwig, BM Devitt, KE Webster. Knee extensor strength and hop test performance following anterior cruciate ligament reconstruction. Knee 2019;26(1):149-54.

35. Sekiya I, T Muneta, T Ogiuchi, K Yagishita, H Yamamoto. Significance of the single-legged hop test to the anterior cruciate ligament-reconstructed knee in relation to muscle strength and anterior laxity. Am J Sports Med 1998;26(3):384-8.

36. Wellsandt E, MJ Failla, L Snyder-Mackler. Limb symmetry indexes can overestimate knee function after anterior cruciate ligament injury. J Orthop Sports Phys Ther 2017;47(5):334-8.

37. Lanshammar K, EL Ribom. Differences in muscle strength in dominant and non-dominant leg in females aged 20-39 years-a population-based study. Phys Ther Sport 2011;12(2):76-9.

\section{Tables}

Table 1. Participant data 


\begin{tabular}{|ll|}
\hline Sex (male/female) & $15 / 32$ \\
\hline Age at testing $(\mathrm{y})$ & $20.0(7.0)$ \\
\hline Height $(\mathrm{cm})$ & $165.0 \pm 0.1$ \\
\hline Weight $(\mathrm{kg})$ & $60.1 \pm 9.1$ \\
\hline Body mass index $\left(\mathrm{kg} / \mathrm{m}^{2}\right)$ & $22.0 \pm 2.1$ \\
\hline Involved limb (left/right) & $28 / 19$ \\
\hline Dominance of involved limb (dominant/non-dominant) & $23 / 24$ \\
\hline Modified Tegner Activity Scale score before injury & $7.0(2.0)$ \\
\hline Days from injury to reconstruction & $55.0(60.0)$ \\
\hline Autograft (hamstring tendon/gracilis + hamstring tendons) & $44 / 3$ \\
\hline Meniscus treated/non-treated & $32 / 15$ \\
\hline Days from reconstruction to testing & $185.0 \pm 16.4$ \\
\hline Sports involved in (basketball/soccer/volleyball/badminton/tennis/frisbee) & $9 / 16 / 12 / 8 / 1 / 1$ \\
\hline
\end{tabular}

Values are mean \pm standard deviation or median (interquartile range). All other values are presented as number of patients or limbs.

Table 2. One-leg hop distance and knee strength

\begin{tabular}{|lll|}
\hline & Involved limb & Uninvolved limb \\
\hline Anterior OLH (\% height) & $64.5(22.8)$ & $76.0(25.7)$ \\
\hline Lateral OLH (\% height) & $46.7(19.0)$ & $57.0(16.8)$ \\
\hline Medial OLH (\% height) & $53.3(19.6)$ & $57.3(18.1)$ \\
\hline Total OLH (\% height) & $165.6(63.0)$ & $188.1(52.2)$ \\
\hline KES 60\% $/ \mathrm{s}(\mathrm{Nm} / \mathrm{kg})$ & $2.0(0.6)$ & $2.4(0.8)$ \\
\hline KES $180 \% / \mathrm{s}(\mathrm{Nm} / \mathrm{kg})$ & $1.4(0.4)$ & $1.7(0.4)$ \\
\hline KFS $60 \% / \mathrm{s}(\mathrm{Nm} / \mathrm{kg})$ & $1.1(0.3)$ & $1.2(0.3)$ \\
\hline KFS $180 \% / \mathrm{s}(\mathrm{Nm} / \mathrm{kg})$ & $0.8(0.3)$ & $0.9(0.3)$ \\
\hline
\end{tabular}

Values are median (interquartile range). OLH, one-leg hop distance; Total, sum of hop distances in 3 directions; KES, knee extension strength; KFS, knee flexion strength.

Table 3. Spearman's rank correlation coefficients between one-leg hop distance and knee strength 


\begin{tabular}{|c|c|c|c|c|}
\hline \multirow[t]{2}{*}{ Involved limb } & \multicolumn{4}{|l|}{$\mathrm{OLH}$} \\
\hline & Anterior & Lateral & Medial & Total \\
\hline KES $60 \% / \mathrm{s}$ & 0.51 ** & $0.52^{\star *}$ & $0.55^{\star \star}$ & $0.54^{\star *}$ \\
\hline KES $180^{\circ} / \mathrm{s}$ & $0.62^{\star \star}$ & $0.65^{\star \star}$ & $0.59 * \star$ & $0.65^{\star \star}$ \\
\hline KFS $60 \%$ s & $0.54^{\star \star}$ & $0.53^{\star *}$ & $0.48 * \star$ & $0.55^{\star \star}$ \\
\hline KFS $180^{\circ} / \mathrm{s}$ & $0.57 \star \star$ & $0.58 * \star$ & $0.54 \star \star$ & $0.59 * *$ \\
\hline \multirow[t]{2}{*}{ Uninvolved limb } & \multicolumn{4}{|l|}{$\mathrm{OLH}$} \\
\hline & Anterior & Lateral & Medial & Total \\
\hline KES $60 \% / \mathrm{s}$ & $0.61^{\star *}$ & $0.58 * \star$ & $0.64^{\star \star}$ & $0.64^{\star *}$ \\
\hline KES $180^{\circ} / \mathrm{s}$ & $0.53^{\star *}$ & $0.51^{\star \star}$ & $0.59 * \star$ & $0.57 * *$ \\
\hline KFS $60 \%$ s & $0.53^{\star *}$ & $0.56^{\star \star}$ & $0.54^{\star \star}$ & $0.57 * *$ \\
\hline KFS $180^{\circ} / \mathrm{s}$ & $0.51^{\star \star}$ & $0.62^{\star \star}$ & $0.60 * \star$ & $0.61^{* *}$ \\
\hline
\end{tabular}

OLH, one-leg hop distance; Total, sum of hop distances in 3 directions; KES, knee extension strength; KFS, knee flexion strength ${ }^{\star *} P<0.01$

\section{Figures}


Patients who underwent ACLR

from May 2015 to July 2019

$\mathrm{N}=276$

Exclusions

- Past experience of ACLR

- Past experience of contralateral ACL injury

$\mathrm{n}=15$

$\mathrm{n}=1$

- Past experience of meniscus surgery

$\mathrm{n}=4$

- Past experience of low back or lower limb injury

that affected physical function

$\mathrm{n}=3$

- Underwent reconstruction of other ligaments of the knee in addition to ACL

- Hoped to return to high-contact sports, martial arts, striking sports, and snow and ice sports

- Unable to visit hospital after ACLR due to social reasons

$\mathrm{n}=134$

$\mathrm{n}=3$

- Does not participate in sports for social reasons after ACLR

$\mathrm{n}=3$

- Had comorbidities that hindered a return to sports

$\mathrm{n}=2$

- Younger than 16 years old at testing

$\mathrm{n}=6$

- Older than 40 years old at testing

$\mathrm{n}=41$

- Unable to complete all questionnaires and physical function tests on the same day between 170 and 240 days after ACLR

- Had missing values

$\mathrm{n}=2$

$\mathrm{n}=4$

Completed measurement

$\mathrm{n}=47$

\section{Figure 1}

Flowchart of patients $\mathrm{ACL}$, anterior cruciate ligament; $\mathrm{ACLR}$, anterior cruciate ligament reconstruction 